\title{
Valoración del Procesamiento LéXico de niños Escolares con Desarrollo Típico de Lenguaje*
}

\author{
Gipsy de la Peña Cruz ${ }^{* *}$ \\ Rossana Gómez Campos*** \\ Marco Cossio-Bolaños ${ }^{* * *}$
}

\section{Resumen}

El objetivo del estudio fue analizar el procesamiento léxico de niños con desarrollo típico del lenguaje. En este estudio descriptivo-explicativo se aplicó un instrumento de 30 palabras para medir el procesamiento léxico a partir de la lectura de la frecuencia de palabras (ruta directa) y la lectura de seudopalabras (ruta indirecta). Los resultados muestran que los niños de ambos sexos, hasta los 11 años reconocen con facilidad las palabras de alta frecuencia (AF) en relación a las palabras de baja frecuencia (BF). A los 12 años existe un equilibrio entre la frecuencia de ambos tipos de palabras; sin embargo, no hubo diferencias en la comparación entre seudopalabras, además el reconocimiento de palabras por la vía directa evidenció un mejor rendimiento en relación a la vía indirecta. La edad cronológica es una variable determinante para alcanzar mejores resultados sobre la lectura de palabras de BF y seudopalabras de alta y baja frecuencia.

Palabras claves: Procesamiento léxico, lectura de palabras, escolares, edad.

\section{Lexical Processing Evaluation of School Children with Typical Language Development}

\begin{abstract}
The aim of the study was to analyze the lexical processing of children with typical language development. In this study descriptive and explanatory words an instrument of 30 was applied to measure the lexical processing from reading the word frequency (direct route) and reading pseudo-words (indirect route). The results show that children of both sexes to 11 years easily recognize the words of high frequency (HF) in relation to the words of low frequency (LF). At age 12 there is a balance between the frequencies of both types of words; however, there was no difference in the comparison between pseudo-words also word recognition by the direct route showed a better performance in relation to the indirect pathway. Chronological age is a determining factor to achieve better results on reading words of $\mathrm{BF}$ and pseudo-words high and low frequency variable.
\end{abstract}

Keywords: Lexical processing, word reading, school, age.

Recibido: 09-06-2015

Aceptado: 22-01-2016

\footnotetext{
Este artículo está asociado al proyecto de investigación "Procesos léxicos en escolares de Curicó" que sirvió de base para desarrollar la tesis de Magíster en el programa del Departamento de Diversidad e Inclusividad Educativa de la Universidad Católica del Maule, Talca, Chile.

** Chilena. Magister en Educación especial y psicopedagogía. Universidad Católica del Maule, Talca, Chile. pesquisadores2010@gmail.com.

*** Peruana. Dra. en Educación Física. Universidad Autónoma de Chile, Talca, Chile. rossaunicamp@ gmail.com

**** Peruano. Dr. En Educación Física. Instituto del deporte Universitario, Universidad Nacional de San Agustín, Arequipa, Perú. Universidad Católica del Maule, Talca, Chile. mcossio1972@hotmail.com
} 


\section{Introducción}

La lectura es una de las actividades más importantes y útiles que el ser humano realiza a lo largo de todas las etapas de la vida. Básicamente, es definida como un proceso complejo y sistemático (Orozco y Pureco, 2007) en el que están inmersos numerosos procesos cognitivos (Golder y Gaonach, 2002), los cuales permiten descifrar el código de la letra impresa para dar posteriormente un significado, el cual se trasluce en comprensión del texto (Valles, 2005).

De hecho, la primera infancia es considerada como una de las etapas cruciales para el aprendizaje de la lectura, ya que para ello se requiere un bagaje de experiencias, tanto lingüísticas como cognitivas, los cuales deben ser estimuladas desde edades muy tempranas, cuya intención es favorecer la elaboración de la información y la construcción de mensajes en forma comprensiva y significativa (Rioseco y Ziliani, 1998).

En general, la actividad lectora requiere del funcionamiento de diversos procesos cognitivos. Para ello, es necesario poner en juego todas las facultades intelectivas, como la memoria, análisis, relación, síntesis, comprensión, inferencia y extrapolación (Orozco y Pureco, 2007), ya que de ellas depende en gran parte el éxito o fracaso lector.

En este sentido, los procesos cognitivos juegan un rol importante dentro del proceso de comprensión y del recuerdo de la información, puesto que esto permite que el lector se ajuste tanto, al texto, como al contexto, transformándolo de esta forma en un proceso activo. De hecho, este proceso es conocido como el procesamiento lector, el cual se inicia a partir de la palabra escrita, sirviendo ésta de estímulo para poner en marcha el proceso visual, cuyo objetivo implica el reconocimiento de la información, el análisis y la emisión de un significado (Fry, 1983). Por lo tanto, dentro del procesamiento lector se pueden distinguir importantes y determinantes procesos que intervienen en la lectura. A este respecto, son varios los estudios (como Cuetos, 2010; Cain, Oakhill \& Bryant, 2004; y Gernsbacher, 1990), que señalan que el procesamiento se realiza en distintos niveles como la percepción de grafemas, decodificación grafema-fonema, reconocimiento de palabras, asignación de funciones sintácticas a las palabras que componen la oración; hasta procesos de mayor nivel, como la integración del significado de las oraciones que componen un texto y la realización de inferencias sobre información implícita. 
En esencia, varios estudios internacionales han dado énfasis en investigar el desarrollo de los procesos cognitivos en niños con antecedentes de retraso y trastorno especifico de lenguaje (SanzTorrent, Andreu, Badia \& Serra, 2010), en alumnos normolectores y con dificultades de aprendizaje (Jiménez, Rodríguez, Guzmán \& García, 2010), en niños con bajo rendimiento en comprensión de lectura (Canet-Juric, Burin, Andrés \& Urquijo, 2013), e inclusive, se ha estudiado los efectos de las variables léxico-semánticas (frecuencia, edad de adquisición e imaginabilidad) sobre el reconocimiento visual de palabras (Alija \& Cuestos, 2006). Sin embargo, poca importancia se le ha dado al estudio del procesamiento léxico en escolares de 7 a 12 años, específicamente en niños que no presenten trastornos específicos del lenguaje, puesto que esta temática tiene un valor funcional en el reconocimiento de la palabra, constituyéndose como un indicador importante para el desarrollo de la adquisición de la lectura en los niños. En este sentido, estudiar la ruta directa visual o léxica (ortográfica) por medio de la lectura de palabras y la ruta indirecta fonológica o subléxica (grafema-fonema) por medio de lectura de seudopalabras en función de la edad y sexo, puede proporcionar información valiosa respecto al conocimiento léxico en términos de familiaridad y disparidad, y de reconocimiento y memoria fonológica en niños en edad escolar. En ese contexto, durante el desarrollo del estudio se pretende dar respuesta a las siguientes interrogantes: a) ¿Cuál es el nivel en que se encuentran los escolares pertenecientes a un colegio particular subvencionado de la provincia de Curicó respecto al procesamiento léxico? y b) ¿La edad cronológica afectará la vía léxica y/o subléxica en los escolares pertenecientes a un colegio particular subvencionado de la provincia de Curicó?

\section{Material y métodos}

Elestudio se propone, como objetivo general, analizar el procesamiento léxico de niños escolares con desarrollo típico del lenguaje perteneciente a una escuela particular subvencionada de la provincia de Curicó (Chile). Adicionalmente, los objetivos específicos tienen que ver con a) Indagar el reconocimiento de lectura palabras (ruta directa) y seudopalabras (ruta indirecta) de escolares de 7 a 12 años de ambos sexos y b) determinar si la edad cronológica influye en la lectura de palabras y lectura de pseudopalabras. 


\subsection{Tipo de estudio y muestra}

El estudio es descriptico-explicativo. Se estudiaron 180 niños de 7,0 a 12,0 años de un establecimiento educacional de zona urbana de la Comuna de Curicó (Chile). Los niños fueron seleccionados de forma probabilística (estratificada por afijación proporcional), siendo el Universo de 800 alumnos (410 niñas y 390 niños). Se determinó el tamaño de la muestra al 95\% IC, resultando seleccionados 180 alumnos (22,5\%), donde $90(11,25 \%)$ son niñas y $90(11,25 \%)$ son niños.

Todos los niños estudiados fueron alumnos de un establecimiento educacional particular (subvencionado), cuyas características consideradas se pueden observar en la tabla 1 . Se incluyeron a todos los escolares cuyos apoderados y / o padres responsables autorizaron la ficha de consentimiento informado, a los que se consideró como alumnos con desarrollo típico de lenguaje y a los que asistieron al día de la evaluación. Se excluyeron a los escolares que se encontraban por debajo de 7,0 años y superior a 12 años de edad y a los que participaban en el programa de integración escolar. Todo el proceso se efectuó de acuerdo a las observaciones del comité de ética local.

Tabla 1.

Características de los escolares estudiados.

\begin{tabular}{lll}
\hline Variables & $\mathbf{f i}$ & $\%$ \\
\hline Sexo: & & \\
\hline Masculino & 90 & 50 \\
\hline Femenino & 90 & 50 \\
\hline Total & 180 & 100 \\
\hline Lugar de procedencia: & & \\
\hline Zona urbana & 12 & 6,3 \\
\hline Zona rural & 178 & 93,7 \\
\hline Total & 190 & 100 \\
\hline Condición socioeconómica: & & \\
\hline Media & 107 & 56,3 \\
\hline Baja & 83 & 43,7 \\
\hline Total & 190 & 100 \\
\hline
\end{tabular}




\subsection{Procedimientos}

La edad decimal se determinó por medio del registro del día, mes y año de nacimiento y el día, mes y año de la evaluación. Todo el proceso de evaluación se llevó a cabo por un solo evaluador con amplia experiencia. Las evaluaciones se efectuaron en el transcurso de la mañana de 8.30 am a 10 am durante los meses de octubre y noviembre del 2013.

Para evaluar el proceso léxico, se utilizó la técnica de la encuesta, cuyo instrumento aplicado fue una parte de la Batería de evaluación de los procesos lectores (PROLEC-R) desarrollado por Cuetos, Rodríguez, Ruano \& Arribas, 2008. Se consideró dos indicadores: a) Lectura de palabras (Palabras de alta frecuencia PAF y palabras de baja frecuencia $\mathrm{PBF}$ ) y b) Lectura de seudopalabras (Seudopalabras de alta frecuencia SPAF y seudopalabras de baja frecuencia SPBF).

Para desarrollar las tareas, se han seleccionado un listado de 5 palabras para cada sub-indicador $(\mathrm{CV}, \mathrm{VC}, \mathrm{CCV}, \mathrm{CVV}, \mathrm{CVVC}$ y CCVC), totalizando 30 palabras para cada sub-indicador: PAF, PBF, SPAF y SPBF. La tabla 2 muestra la operacionalización de la variable estudiada con sus respectivos indicadores y sub-indicadores. La valoración tuvo un puntaje de 0,1 y 2 puntos (bueno $=2$ puntos, regular $=1$ punto, $y$ malo $=0$ puntos). A todos los alumnos se les mostró una figura en el que debían reconocer la frecuencia de palabras y las seudopalabras. En el proceso se les pedía leer en voz alta las 30 palabras, de una en una, conforme se les iba presentando cada palabra (sentado frente al alumno). En ese transcurso, el evaluador emitía un juicio de valor expresado en bueno, regular y malo, según la lectura que efectuaba el alumno. El procedimiento tuvo una duración promedio de 5 minutos por estudiante. Todo el proceso se llevó a cabo en una sala de aula, entre el evaluador y el alumno, manteniendo las condiciones mínimas de evaluación (adecuada visibilidad y baja presencia de ruidos extraños). Previamente, antes de iniciar la evaluación se les explicó el procedimiento a todos los alumnos, indicándoles la forma de aplicar el instrumento. 
Tabla 2.

Operacionalización de la variable estudiada.

\begin{tabular}{|c|c|c|c|c|}
\hline Variable & Indicadores & Sub-indicadores & Ítems & Cantidad \\
\hline \multirow{24}{*}{$\begin{array}{l}\text { Procesamiento } \\
\text { Léxico }\end{array}$} & \multirow{12}{*}{$\begin{array}{l}\text { 1. Lectura de } \\
\text { palabras }\end{array}$} & \multirow{6}{*}{$\begin{array}{l}\text { 1.1. Palabras de alta } \\
\text { frecuencia (PAF) }\end{array}$} & $\mathrm{CV}$ & 5 \\
\hline & & & $\mathrm{VC}$ & 5 \\
\hline & & & $\mathrm{CCV}$ & 5 \\
\hline & & & $\mathrm{CVV}$ & 5 \\
\hline & & & CVVC & 5 \\
\hline & & & $\mathrm{CCVC}$ & 5 \\
\hline & & \multirow{6}{*}{$\begin{array}{l}\text { 1.2. Palabras de baja } \\
\text { frecuencia (PBF) }\end{array}$} & $\mathrm{CV}$ & 5 \\
\hline & & & $\mathrm{VC}$ & 5 \\
\hline & & & $\mathrm{CCV}$ & 5 \\
\hline & & & $\mathrm{CVV}$ & 5 \\
\hline & & & CVVC & 5 \\
\hline & & & CCVC & 5 \\
\hline & \multirow{12}{*}{$\begin{array}{l}\text { 2. Lectura de } \\
\text { seudopalabras }\end{array}$} & \multirow{8}{*}{$\begin{array}{l}\text { 2.1. Palabras de alta } \\
\text { frecuencia (PAF) }\end{array}$} & $\mathrm{CV}$ & 5 \\
\hline & & & VC & 5 \\
\hline & & & $\mathrm{CCV}$ & 5 \\
\hline & & & $\mathrm{CVV}$ & 5 \\
\hline & & & CVVC & 5 \\
\hline & & & CCVC & 5 \\
\hline & & & $\mathrm{CV}$ & 5 \\
\hline & & & VC & 5 \\
\hline & & \multirow{4}{*}{$\begin{array}{l}\text { 2.2. Palabras de baja } \\
\text { frecuencia (PBF) }\end{array}$} & $\mathrm{CCV}$ & 5 \\
\hline & & & $\mathrm{CVV}$ & 5 \\
\hline & & & CVVC & 5 \\
\hline & & & CCVC & 5 \\
\hline
\end{tabular}

Leyenda: $\mathrm{CV}$ consonante vocal; VC vocal, consonante;CCV consonante, consonante, vocal; CVV consonante, vocal, vocal; CVVC consonante, vocal, vocal, consonante; CVVC consonante, vocal, vocal, consonante.

El instrumento utilizado mostró una alta capacidad de reproductibilidad, donde el coeficiente de consistencia interna de alpha de Cronbach para los niños fue de 0,75, para las niñas de 0,86, y para ambos sexos de 0,83 . 
Los datos como el lugar de procedencia y la clasificación socioeconómica fueron obtenidos de la ficha de matrícula de cada alumno. Tales datos y fichas fueron proporcionados por el Director de la Institución Educativa.

El estatus léxico se determinó a partir de la diferencia entre la frecuencia de palabras (PAF+PBF) y las seudopalabras (SPAF+SPBF). Por ejemplo, en niños de 7,0 años, la sumatoria de PAF+PBF fue de 114,7 puntos (ver tabla 4).

\subsection{Estadística}

La distribución normal fue verificada a través de la prueba Kolmogorov Smirnov. Los datos fueron analizados a partir de la estadística descriptiva de media, desviación estándar, frecuencias y porcentajes. Las diferencias entre sexos se verificaron por medio de test $t$ para muestras independientes. Las comparaciones entre palabras (intragrupos) se efectuó por medio del test t para muestras relacionadas. La relación de causa efecto se determinó por medio de análisis de regresión simple (stepwise), considerando la edad como variable independiente y la lectura de PAF, PBF, SPAF y SPBF como dependientes. En todos los casos se utilizó $\mathrm{p}<0,05$. Todos los datos fueron procesados mediante el programa Sigma Estat 8,0 y planillas de Excel.

\section{Resultados}

Los indicadores que comprenden el procesamiento léxico se observan en la tabla 3. En cuanto a la lectura de palabras, hubo diferencias entre PAF vs PBF desde los 7,0 hasta los 11,9 años, y en ambos sexos. Respecto a las comparaciones entre PAF vs SPAF y PAF vs SPBAF, se verificó diferencias significativas en ambos sexos desde los 7,0 años hasta los 10,9 años $(p<0,05)$. Sin embargo, no hubo diferencias a los 12 años en ambos sexos. A su vez, cuando se comparó entre sub-indicadores entre seudopalabras (SPAF vs SPBAF) no se evidenció diferencias significativas $(\mathrm{p}>0,05)$. 
Tabla 3.

Valores medios y DE de los indicadores que comprenden el procesamiento léxico en niños por edad y sexo.

\begin{tabular}{|c|c|c|c|c|c|c|c|c|c|}
\hline \multirow[b]{3}{*}{ Edad } & \multirow[b]{3}{*}{$\mathrm{n}$} & \multicolumn{4}{|c|}{ Ruta Directa (visual) } & \multicolumn{4}{|c|}{$\begin{array}{l}\text { Ruta Indirecta } \\
\text { (fonológica) }\end{array}$} \\
\hline & & \multicolumn{2}{|l|}{ PAF } & \multicolumn{2}{|l|}{ PBF } & \multicolumn{2}{|l|}{ SPAF } & \multicolumn{2}{|l|}{ SPBF } \\
\hline & & $X$ & $\mathrm{DE}$ & $X$ & $\mathrm{DE}$ & $X$ & $\mathrm{DE}$ & $X$ & $\mathrm{DE}$ \\
\hline \multicolumn{10}{|l|}{ Niños } \\
\hline $\begin{array}{l}7,0- \\
7,9\end{array}$ & 15 & 58,9 & 1,4 & 55,8 & $3,4^{*}$ & 54,1 & $3,6+$ & 53,4 & $3,4++$ \\
\hline $\begin{array}{l}8,0- \\
8,9\end{array}$ & 15 & 58,7 & 1,9 & 56.6 & $2,4^{*}$ & 53,1 & $6,4+$ & 52,6 & $3,2++$ \\
\hline $\begin{array}{l}9,0- \\
9,9\end{array}$ & 15 & 59,6 & 1,2 & 57,0 & $2,8^{*}$ & 55,3 & $3,8+$ & 53,8 & $5,0++$ \\
\hline $\begin{array}{l}10,0- \\
10,9\end{array}$ & 15 & 59,7 & 0,6 & 58.8 & $1,4^{*}$ & 55,9 & $3,4+$ & 57,6 & $6,5+t$ \\
\hline $\begin{array}{l}11,0- \\
11,9\end{array}$ & 15 & 59,6 & 0,8 & 58.8 & $1,3^{*}$ & 56,9 & $1,6+$ & 57,1 & 2,3 \\
\hline $\begin{array}{l}12,0- \\
12,9\end{array}$ & 15 & 59,7 & 1,0 & 59,5 & 1,1 & 57,5 & $2,1 \dagger$ & 58,1 & 2,6 \\
\hline Todos & 90 & 59,4 & 1,3 & 57,7 & $2,6^{*}$ & 55,5 & $4,0 \dagger$ & 55,4 & $4,6+t$ \\
\hline \multicolumn{10}{|l|}{ Niñas } \\
\hline $\begin{array}{l}7,0- \\
7,9\end{array}$ & 15 & 58,1 & 3,1 & 57,8 & $3,3^{*}$ & 50,9 & 9,0 & 51,6 & $8,7++$ \\
\hline $\begin{array}{l}8,0- \\
8,9\end{array}$ & 15 & 59,5 & 0,8 & 57,3 & $2,6^{*}$ & 55,1 & 4,6 & 54,5 & $4,7++$ \\
\hline $\begin{array}{l}9,0- \\
9,9\end{array}$ & 15 & 59,7 & 0,7 & 57,9 & $2,1^{*}$ & 55,2 & 3,8 & 54,9 & $4,9++$ \\
\hline $\begin{array}{l}10,0- \\
10,9\end{array}$ & 15 & 59,8 & 0,8 & 58,5 & $1,8^{*}$ & 56,1 & 2,4 & 56,3 & $3,3++$ \\
\hline $\begin{array}{l}11,0- \\
11,9\end{array}$ & 15 & 59,7 & 1,0 & 58,4 & $1,4^{*}$ & 57,5 & 2,8 & 57,2 & 1,7 \\
\hline $\begin{array}{l}12,0- \\
12,9\end{array}$ & 15 & 60,0 & 0,0 & 59,0 & 1,7 & 57,8 & 2,7 & 58,5 & 1,8 \\
\hline Todos & 90 & 59,5 & 1,4 & 58,2 & $2,3^{*}$ & 55,4 & 5,3 & 55,4 & 5,3 \\
\hline
\end{tabular}

Leyenda: PAF Alta Frecuencia, PBF Baja Frecuencia, DE, desviación estándar, *=PAF vs $\mathrm{PBF}, \mathrm{t}=\mathrm{PAF}$ vs SPAF $\mathrm{y}+\mathrm{t}=\mathrm{PBF} \vee \mathrm{SPBF}(\mathrm{p}<0,05)$. 
En cuanto al estatus léxico, los resultados muestran que la frecuencia de palabras (PAF+PBF) evidenció altos valores en relación a las seudopalabras (SPAF+SPBF) en todas las edades y en ambos sexos.

Tabla 4.

Valores medios y DE del Estatus léxico de niños por edad y sexo.

\begin{tabular}{|c|c|c|c|c|c|}
\hline \multirow[b]{2}{*}{ Edad } & \multirow[b]{2}{*}{$\mathrm{n}$} & \multicolumn{2}{|c|}{$\begin{array}{l}\text { (Ruta directa) Frecuencia de } \\
\text { Palabras } \\
\text { AF+BF }\end{array}$} & \multicolumn{2}{|c|}{$\begin{array}{l}\text { (Ruta indirecta) } \\
\text { Seudopalabras } \\
\text { AF+BF }\end{array}$} \\
\hline & & $X$ & $\mathrm{DE}$ & $x$ & $\mathrm{DE}$ \\
\hline \multicolumn{6}{|l|}{ Niños } \\
\hline $7,0-7,9$ & 15 & 114,7 & 4,3 & 107,5 & $5,9^{*}$ \\
\hline $8,0-8,9$ & 15 & 115,3 & 3,7 & 105,7 & $9,3^{*}$ \\
\hline $9,0-9,9$ & 15 & 116,6 & 3,2 & 109,1 & $8,4^{*}$ \\
\hline $10,0-10,9$ & 15 & 118,5 & 1,9 & 113,6 & $8,8^{*}$ \\
\hline $11,0-11,9$ & 15 & 118,4 & 1,5 & 114,1 & $3,3^{*}$ \\
\hline $12,0-12,9$ & 15 & 119,2 & 2,0 & 115,6 & $4,3^{*}$ \\
\hline Todos & 90 & 119,2 & 2,1 & 115,3 & $4,3^{*}$ \\
\hline \multicolumn{6}{|l|}{ Niñas } \\
\hline $7,0-7,9$ & 15 & 115,8 & 6,3 & 102,5 & $17,1^{*}$ \\
\hline $8,0-8,9$ & 15 & 116,8 & 3,0 & 109,6 & $8,7^{\star}$ \\
\hline $9,0-9,9$ & 15 & 117,7 & 2,4 & 110,1 & $8,3^{*}$ \\
\hline $10,0-10,9$ & 15 & 118,3 & 2,0 & 112,5 & $5,1^{*}$ \\
\hline $11,0-11,9$ & 15 & 118,6 & 2,0 & 114,7 & $3,9^{*}$ \\
\hline $12,0-12,9$ & 15 & 119,0 & 1,7 & 116,3 & $3,6^{*}$ \\
\hline Todos & 90 & 117,7 & 3,4 & 110,8 & $9,8^{*}$ \\
\hline
\end{tabular}

Leyenda: AF Alta Frecuencia, BF Baja Frecuencia, DE, desviación estándar, ${ }^{*}=(\mathrm{p}<0,05)$.

La tabla 5 muestra los valores de regresión estadística (stepwise), donde la edad es considerada como variable independiente y los indicadores del proceso lector (PAF, PBF, SPAF y SPBF) como variables dependientes. En ambos sexos se observa que la edad no afecta sobre el indicador denominado lectura de PAF, a pesar de existir una modesta correlación en ambos sexos (hombres $r=0,28$ y niñas $r=0,27$ ). Sin embargo, sucede lo contrario para los demás indicadores, donde la edad explica las $\mathrm{PBF}$, SPAF y SPBF en un 12 a 25\% en los niños y en un 11 a $17 \%$ en las niñas. 
Tabla 5.

Valores de regresión (stepwise) de los indicadores del procesamiento léxico a partir de la edad cronológica.

\begin{tabular}{lllllll}
\hline Variables & $\mathrm{B}$ & $\mathrm{C}$ & $\mathrm{R}$ & $\mathrm{R}^{2}$ & $\mathrm{EEM}$ & $\mathrm{P}$ \\
\hline Niños & & & & & & \\
PAF & 0,21 & 57,4 & 0,28 & 0,01 & 1,22 & 0,77 \\
PBF & 0,76 & 50,5 & 0,51 & 0,25 & 2,21 & $<0,001$ \\
SPAF & 0,83 & 47,5 & 0,36 & 0,12 & 3,77 & $<0,001$ \\
SPBF & 1,16 & 44,4 & 0,44 & 0,18 & 4,12 & $<0,05$ \\
Niñas & & & & & & \\
PAF & 0,40 & 54,9 & 0,27 & 0,06 & 2,21 & 0,71 \\
PBF & 0,30 & 56,5 & 0,34 & 0,11 & 1,50 & $<0,001$ \\
SPAF & 1,30 & 43,4 & 0,41 & 0,16 & 4,87 & $<0,001$ \\
SPBF & 1,30 & 43,0 & 0,43 & 0,17 & 4,77 & $<0,001$ \\
\hline
\end{tabular}

4. Leyenda: $ß=$ beta, $C=$ constante, $\mathrm{EEM}=$ Erros estándar de medida.

\section{Conclusiones y proyecciones}

Dentro del procesamiento léxico se puede distinguir importantes procesos que intervienen durante la lectura. Básicamente, se sugiere conocer dos variables según lo propuesto por Cuetos et al. (2008) y analizar la existencia de doble ruta léxica (Coltheart, 1981), en el que se destaca la lectura de palabras (ruta léxica, visual o directa) y la lectura de seudopalabras (ruta indirecta, subléxica o fonológica). A este respecto, el presente estudio abordó, inicialmente, el procesamiento léxico de escolares con desarrollo típico de lenguaje en el que se valoró ambas rutas de acceso a la lectura y, seguidamente, el efecto de la edad sobre los indicadores del procesamiento léxico.

Como era de esperar, respecto a la ruta léxica, los resultados del estudio muestran que los escolares de ambos sexos reconocen con más facilidad las palabras de AF en relación a las de BF hasta los 11 años; sin embargo, a los 12 años no se observaron diferencias, por lo que se supone que los niños a esta edad reconocen con más facilidad las palabras de baja frecuencia, debido a las múltiples habilidades fonológicas que han ido adquiriendo a lo largo de los años. En este sentido, González y Cuetos (2009) destacan que las palabras más frecuentes son las que generalmente 
se aprenden antes y las de BF son las que se aprenden a una edad más tardía. De hecho, en este estudio se evidenció que a partir de los 12 años es posible que exista un equilibrio en el reconocimiento entre palabras de $\mathrm{AF}$ y BF, que por lo general es de esperar en términos de rendimiento, aunque algunas veces se suele presentar el denominado efecto espejo, en el que las palabras de BF presentan una alta tasa de aciertos en relación a las palabras de AF. Para ello, se sugiere efectuar más estudios con el propósito de verificar dicho efecto, abarcando escolares de más edades y de diversos contextos socioculturales, puesto que es ampliamente conocido que niños que viven en ambientes socioculturales pobres tienden a tener un desempeño lector más limitado y mayores problemas de aprendizaje en la lectura (Massaro, 2002).

En efecto, varios estudios hacen hincapié en el uso de las palabras de $\mathrm{AF}$, destacando que se suelen reconocer en menos tiempo en relación a las palabras de BF (Grainger, 1990; Strain et al., 1995; Monsalve y Cuetos, 2001), dado que las palabras de AF se destacan por ser las más familiares, las más concretas y por adquirirse a edades tempranas (Alija y Cuetos, 2006). Inclusive, tales palabras a menudo se suelen reconocer al instante y generalmente se asocian casi mecánicamente con su significado (Álvarez y González, 1996). Sin embargo, las palabras de BF presentan menos enlaces asociativos (González y Cuetos, 2009), que por lo general son difíciles de recordar y básicamente presentan un mayor porcentaje de errores, por lo tanto, requieren de un mayor repertorio de palabras, el cual se irá adquiriendo en función de la edad y el ambiente sociocultural en el que se desenvuelvan.

Respecto a la comparación entre seudopalabras de $\mathrm{AF}$ y $\mathrm{BF}$, no se evidenciaron diferencias significativas; sin embargo, cuando se comparó entre las palabras de AF vs SPAF y palabras de BF vs SPBF, la frecuencia de palabras mostró valores superiores en todas las edades y en ambos sexos. Esto demuestra que los escolares estudiados hacen uso de la ruta directa (léxica), donde básicamente predomina el reconocimiento de las palabras de AF y BF sobre las seudopalabras. De hecho, este patrón se observa hasta los 10 años, puesto que a los 11 y 12 años en ambos sexos no hubo diferencias, por lo que el reconocimiento de palabras por la vía directa e indirecta mostró similar nivel de rendimiento. Por lo tanto, se estima que a partir de los 10 años en adelante, los niños de este estudio son capaces de reconocer las palabras por ambas vías léxicas. 
Por otro lado, en términos de lexicalidad (estatus léxico), se observó que la lectura de palabras $(\mathrm{PAF}+\mathrm{PBF})$ presentó valores superiores en comparación con las seudopalabras (SPBF+SPBF). De hecho, algunos estudios han informado la existencia de déficits en la repetición de seudopalabras (Bishop, North \& Donland, 1996; Botting \& ContiRamnden, 2001), esto en vista de que los niños desde las primeras etapas de la adquisición lectora aún no están acostumbrados a usar las formas fonológicas desconocidas, sobre todo a edades tempranas, puesto que para ello se requiere de mayor experiencia lectora y que esto se irá ganando con el transcurso del tiempo y dependerá del tipo de estrategia de enseñanza utilizada; inclusive, la atención es un factor indispensable para lograr una adecuada decodificación de los estímulos y comprensión de textos (Rosselli, Matute y Ardilla, 2006). En este sentido, autores como Domínguez y Cuetos (1992) consideran que las diferencias entre frecuencia de palabras y seudopalabras son más notorias en las personas que recién están aprendiendo a leer, por lo que la repetición de seudopalabras constituye una medida de la capacidad de la memoria fonológica (Gathercole et al., 1999), el cual es clave para los estados iniciales del aprendizaje de la lectura en un sistema alfabético (Álvarez y González, 1996) como el español.

En esencia, tanto la ruta directa (léxica, visual), como la ruta indirecta (subléxica o fonológica) funcionan interactuando de manera continua, de tal forma que el reconocimiento de palabras escritas y la lectura de palabras en voz alta es el resultado de la participación de ambas vías (Coltheart, 1981). Por lo tanto, el papel que desempeña cada una de ellas depende de cada palabra en concreto y de cada lector en particular (Cuetos, Domínguez, 2002), por lo que el rendimiento que se pueda mostrar en términos de procesamiento léxico dependerá básicamente de factores socioambientales, como el nivel de estimulación, el ambiente sociocultural, la estrategia y la edad de iniciación, aunque hay quienes cuestionan la existencia de una doble ruta en lengua española (Ardila, 1998), destacando una sola ruta denominada fonológica o indirecta, la que sería la más importante.

En cuanto al efecto de la edad sobre los indicadores del procesamiento léxico, se verificó que en ambos sexos la edad no afecta el rendimiento de las palabras de AF; sin embargo, sí es determinante para el reconocimiento de las palabras de $\mathrm{BF}$ y las seudopalabras de $\mathrm{AF}$ y $\mathrm{BF}$, respectivamente. $\mathrm{El}$ 
poder de explicación para los tres indicadores es mayor en los niños (12$25 \%$ ) que en las niñas (11-17\%), por lo que se estima que la edad cronológica es determinante para en el reconocimiento de palabras desconocidas y las que no existen dentro del vocabulario de la lengua hispana, puesto que a medida que la edad avanza los niños van adquiriendo nuevas palabras para su vocabulario y, al mismo tiempo, van ganando experiencia en el reconocimiento de nuevas palabras existentes y no existentes.

Evidentemente, respecto a esta situación los estudios son controversiales, dado que algunas investigaciones han demostrado que la edad es determinante (Nagy, et al., 1989; Alija y Cuetos, 2006), pero otros estudios han verificado lo contrario (Rubín, 1980; Paivio, 1986). Por lo tanto, a partir de estos hallazgos, se sugiere desarrollar más estudios, sobre todo de corte longitudinal. Además de lo anterior, es necesario ampliar el rango de edad y valorar la latencia sobre el reconocimiento de palabras, con lo cual es posible verificar tales resultados.

En esencia, cabe resaltar que durante el estudio no se pudo valorar el tiempo de latencia en el reconocimiento de palabras en general, además que la velocidad de lectura hubiera sido una variable relevante para analizar de mejor forma los resultados obtenidos. Al respecto, se sugiere para futuros estudios controlar tales variables y diseñar estudios longitudinales para verificar los cambios del procesamiento léxico en función de la edad y sexo, respectivamente.

Se concluye que los niños de ambos sexos hasta los 11 años reconocen con facilidad las palabras de AF en relación a las palabras de BF. Posteriormente, a los 12 años se observó un equilibrio entre la frecuencia de ambos tipos de palabras; sin embargo, no hubo diferencias en la comparación entre seudopalabras, además que el reconocimiento de palabras por la vía directa evidenció un mejor rendimiento en relación a la vía indirecta; inclusive, la edad cronológica es una variable determinante para alcanzar mejores resultados sobre la lectura de palabras de BF y seudopalabras de alta y baja frecuencia, respectivamente. Estos resultados sugieren que los niños estudiados reconocen las palabras por la vía directa, y que la edad cronológica puede ser considerada como un indicador determinante para predecir la lectura de palabras de BF y seudopalabras de baja y alta frecuencia. 


\section{Referencias Bibliográficas}

Alija, M. y Cuetos, F. (2006). Efectos de las variables léxico-semánticas en el reconocimiento visual de palabras. Psicothema, 18(3), 485-491.

Álvarez Pérez L. y González Castro, P. (1996). Dificultades en la adquisición del proceso lector. Psicothema, 8, 3, 573586.

Ardila, A. (1998). Semantic paralexias in spanish language. Aphasiology, $12,885-900$.

Bishop, D. M. V., North, T., \& Donland, C. (1996). Non-word repetition as a behavioral marker for inhereted language impairment: Evidence from a twin study. Journal of Child Psychology and Psychiatry, 37, 391-403.

Botting, N. \& Conti-Ramsden, G. (2001). Non-word repetition and language development in children with specific language impairment (SLI). International Journal of Language and Communication Disorders, 36, 421-432.

Cain, K., Oakhill, J. \& Bryant, P. E. (2004). Children's reading comprehension ability: Concurrent prediction by working memory, verbal ability, and com-ponent skills. Journal of Educational Psychology, 96, 31-42.

Canet-Juric L, Burin D, Andrés ML. \& Urquijo S. (2013). Perfil cognitivo de niños con rendimientos bajos en comprensión lectora. Anales de Psicología, 29(3), 996-1005.

Coltheart, M. (1981). Disorders of reading and their implications for models of normal reading. Visible Language, 15(3), 245-286.

Cuetos, F., Rodríguez, B., Ruano., \& Arribas, D. (2008). Batería de evaluación de los proceso lectores, Revisada. Madrid, España: Tea Ediciones.

Cuetos, F. (2010). Psicología de la lectura. Octava Edición. Madrid. España: Wolters. 
Domínguez, A. y Cuetos, F. (1992). Desarrollo de las habilidades de reconocimiento de palabras en niños con distinta competencia lectora. Cognitiva, 4, 193-208.

Fry, G.A. (1983). Basic concepts underlying graphical analysis. In Schor CM and Ciuffreda K.J.(Eds.), Vergence Eye Movements: Basic and Clinical Aspects. Boston: Butterworth.

Gathercole, S., Hitch, G. J., Service, E., \& Martin, A.J. (1999). Phonological short term memory and vocabulary development: further evidence on the nature of the relationship. Applied Cognitive Psychology, 13, 65-77.

Gernsbacher, M. A. (1990). Language comprehension as structure building. Hillsdale, NJ: Erlbaum.

Golder, C., \& Gaonach, D. (2002). Leer y comprender psicología de la lectura. Innovación Educativa, 7(39), 78-85.

González, N. y Cuetos F. (2009). Influencia de las variables psicolingüísticas sobre el efecto de generación. Psicológica, 30, 323-342.

Grainger, J. (1990). Word frequency and the neighbourhood frequency effects in lexical decision and word naming. Journal of Memory and Language, 29, 228-244.

Jiménez, J.E., Rodríguez C., Guzmán R. \& García E. (2010). Desarrollo de los procesos cognitivos de la lectura en alumnos normolectores y alumnos con dificultades específicas de aprendizaje. Revista de Educación, 353, 361-383.

Massaro, D.W. (2002). Building and testing models of reading processes. In Pearson PD (Ed.), Handbook of reading research (pp. 11.146). Mahwah, NJ: Lawrence Erlbaum.

Monsalve, A. y Cuetos, F. (2001). Asimetria hemisferica en el reconocimiento de palabras: Efectos de frecuencia e imaginabilidad. Psicothema, 13, 24-28.

Nagy, W., Anderson, R, Schommer, M., Scott, J. \& Stallman, A. (1989). Morphological families in the internal lexicon. Reading Research Quarterly, 24, 262-282. 
Orozco, M. y Pureco, F. (2007). El proceso de la lectura. Ethos educativo, $39,59-70$.

Paivio, A. (1986). Mental representation: A dual-coding approach. New York: Oxford University Press.

Rioseco, R. y Ziliani, M. (1998). Pensamos y aprendemos. Lenguaje y comunicación. Santiago: Andrés Bello.

Rosselli, M., Matute, E., Ardila, A. (2006). Predictores neuropsicológicos de la lectura en español. Rev Neurol, 42(4), 202-210.

Rubin, D. C. (1980). 51 properties of 125 words: A unit analysis of verbal behavior. Journal of Verbal Learning and Verbal Behavior, 19, 736-755.

Sanz-Torrent M., Andreu, L.I., Badia, M., \& Serra M. (2010). El proceso lector en niños con antecedentes de retraso y trastorno específico del lenguaje. Revista de Logopedia, Foniatría y Audiología, 1(30), 23-33.

Strain, E., Patterson, K.E. \& Seidenberg, M.S. (1995). Semantic effects in single-word naming. Journal of Experimental Psychology: Learning, Memory and Cognition, 21(5), 1140-54.

Valles, A. (2005). Comprensión lectora y procesos psicológicos. Liberabit. Revista de Psicología, 11, 49-61. 